\title{
CONSIDERACIONES EN TORNO AL LÉXICO TURÍSTICO EN LA ENSEÑANZA DEL ALEMÁN CON FINES ESPECÍFICOS
}

\author{
María A. Borrueco Rosa
}

\section{INTRODUCCIÓN}

Los estudios de Turismo surgen como una auténtica necesidad ante el fuerte crecimiento del turismo en los años 60, adquiriendo su pleno reconocimiento a partir de 1980, año en el que se configuran como enseñanza universitaria. Se conforman como enseñanza especializada de carácter interdisciplinar integrada por áreas de conocimiento de diferente naturaleza: formación económico-empresarial (Derecho, Economía, Contabilidad, Gestión y Dirección de empresas ... ), formación humanística (Geografía turística, Historia del arte ... ), formación técnica (Gestión de agencias de viajes, Gestión de alojamientos turísticos, Marketing turístico, Turismo rural ... ) y, finalmente, formación en el ámbito de segundas lenguas. Son muchas las razones que justifican la presencia de segundas lenguas como área esencial en la formación de profesionales del turismo. El carácter internacional del fenómeno turístico hace que millones de turistas estén en continuo movimiento visitando lugares fuera de sus fronteras. Estos visitantes demandan un gran número de servicios: alojamiento, restauración, ocio, cultura, etc. y, por tanto, un personal altamente cualificado no sólo a nivel técnico sino también y sobre todo en lo que a la lengua se refiere, una cualificación en idiomas. El crecimiento constante del turismo a nivel mundial, el incremento de la oferta desde todos los rincones del mundo y el mayor nivel de exigencia por parte de los consumidores del producto turístico hacen que el hecho de dominar varias lenguas emerja como una cualidad preponderante, postergada antaño a un segundo nivel.

Por otro lado, y no por ello menos importante, ha de mencionarse la relación empresarial de carácter internacional que deriva de la actividad turística. Muchas actividades y empresas turísticas se apoyan de forma esencial en las relaciones comerciales entre empresas diversas ubicadas en distintos países. Esta creciente actividad requiere la presencia de profesionales con capacidad para comunicarse en varias lenguas, profesionales que de esta forma sirven de puente en la gestión empresarial. Contribuyen así al éxito de numerosas actividades asociadas al fenómeno turístico y relacionadas principalmente con el ocio y la ocupación del tiempo libre. Buena muestra de ello son las numerosas Ferias Internacionales de Turismo de carácter anual como FITUR (Feria Internacional de Turismo) en Madrid o la ITB (Internationale Tourismus-Börse) en Berlín o, por otro lado, la existencia de relaciones 
bilaterales entre grandes touroperadores o incluso la integración de empresas españolas en multinacionales extranjeras.

En este contexto ha sido la lengua inglesa, sin duda alguna, protagonista durante décadas. Pero no de forma exclusiva. La lengua alemana y también la francesa han compartido este protagonismo dada la importancia del turismo alemán y francés en nuestro país. Es así como ya desde los Planes de Estudio de 1963, que facultaban a la obtención del título oficial de Técnico en Empresas Turísticas se contemple la enseñanza del alemán como segunda lengua, presencia que se consolida en la reforma de los estudios superiores de turismo efectuada en 1980 y en los actuales Planes de Estudio de la Diplomatura de Turismo, desarrollados en 1996. La lengua alemana, junto con el francés y el inglés conforman así una parcela muy importante en la formación de los futuros Diplomados en Turismo.

El problema planteado a no pocos docentes es el de cómo abordar la enseñanza de una segunda lengua en el marco de un perfil profesional concreto ${ }^{1}$. Es una tendencia ya generalizada hablar de la enseñanza de la segunda lengua con fines específicos cuando hacemos referencia al alemán empresarial, comercial, científico o jurídico. Pero hasta fecha muy reciente no se produce esta circunstancia respecto a la formación turística. La actitud casi general ha sido ignorar el carácter "específico" y, a lo sumo, integrar en un método de carácter general algunas unidades didácticas que introducían un reducido corpus léxico relacionado con el sector, en la mayoría de los casos referente únicamente al alojamiento turístico y al transporte de viajeros. Pero lo cierto es que los egresados utilizarán la lengua extranjera primordialmente a nivel profesional. Los diplomados en turismo entrarán en contacto directo con el visitante o con el empresario extranjero, debiendo conocer y activar un léxico muy concreto que estará en función del subsector en el que desarrollen su actividad. Es en este contexto en el que se hace patente la carencia de un corpus léxico al que no ha tenido acceso durante su período de formación. Aún así habrá de hacer frente de forma reiterada a situaciones comunicativas específicas que derivan del ejercicio de su actividad laboral. De forma análoga a empresarios, científicos o juristas necesita una formación de carácter específico.

\section{EL LÉXICO ESPECIALIZADO}

Como ya apuntaba en 1969 Trim, estructuralmente no hay muchas diferencias entre una lengua extranjera estándar ${ }^{2}$ de una lengua extranjera con fines específicos, es básicamente el léxico el que establece los caracteres diferenciales. Lógicamente se ha de proceder a la delimitación y sistematización de este corpus. La cuestión a plantear es ¿cómo delimitar este léxico de carácter específico ${ }^{3} \mathrm{Si}$ en la enseñanza de segundas lenguas estándar el

\footnotetext{
${ }^{1}$ Es de destacar el auge que desde los 80 experimenta la enseñanza de segundas lenguas con fines específicos: Véase FLUCK (1992).

${ }^{2}$ Entiéndase aquí como lengua estándar la aplicada en contextos de tipo general no especializados.

${ }^{3}$ Véase la tesis formulada por EGGERT, S. (1991), que referente a la sistematización del léxico alemán como segunda lengua defiende el acercamiento cognitivo al mismo.
} 
criterio de frecuencia es el aplicado, en la enseñanza con fines específicos debe matizarse este criterio. Así por ejemplo en carreras de perfil técnico es generalmente el contenido científico de las materias impartidas el que determina el léxico estudiado en la enseñanza de segundas lenguas, al igual que la metodología que en muchos casos se centra en la lectura de textos científicos ${ }^{4}$. Se potencia así principalmente el dominio pasivo de la lengua. La competencia lingüística de los futuros diplomados en Turismo se aleja absolutamente de esta perspectiva del idioma, pues, a excepción de la correspondencia comercial, utilizará la segunda lengua para comunicarse de forma directa con sus clientes o con otros empresarios. Deducimos así que es sin duda alguna el perfil profesional del diplomado en Turismo el hilo conductor en la tarea de sistematización del léxico. Un análisis en detalle de la futura actividad del egresado facilitará la selección del corpus mencionado. Los factores determinantes de este análisis son dos: por un lado los subsectores en los que habremos de dividir el sector turístico, dada la amplitud que ofrece, y por otro, las actividades profesionales que se desarrollan en cada uno de ellos. Estas actividades delimitarán de forma natural la competencia lingüística requerida, es decir las situaciones comunicativas, e indirectamente el léxico asociado a ellas.

Los principales sectores (LÓPEZ: 1992) que se distinguen son:

\author{
Alojamiento de viajeros / Unterkunft der Reisenden \\ Transporte de viajeros / Beförderung der Reisenden (Transport) \\ Transporte aéreo / Luftverkehr ( Flugverkehr) \\ Transporte por ferrocarril / Schienenverkehr (Bahnverkehr) \\ Transporte marítimo o fluvial / Wasserverkehr (Schiffsverkehr, Seereiseverkehr, Binnenrei- \\ severkehr) \\ Transporte por carretera / Straßenverkehr
}

La organización de viajes y mediación (distribución y venta) / Reiseveranstaltung und Reisevermittlung

Actividades turísticas relacionadas con el ocio y el tiempo libre / Touristische Aktivitäten

En cada uno de estos sectores emergen con entidad propia empresas que desarrollan múltiples actividades relacionadas con cada uno de ellos. Así los diplomados en turismo podrán integrarse en la dirección y gestión por ejemplo de las siguientes empresas:

Relacionadas con el alojamiento turístico podemos citar los hoteles, apartahoteles, pensiones, paradores de turismo (sólo en España), albergues juveniles, casas rurales, etc.

\footnotetext{
${ }^{4}$ Es de destacar la postura defendida por algunos docentes que reivindican precisamente una metodología "no tan específica" en este área. Véanse en este sentido los argumentos expuestos por Aurora Astor y Marta Koller en "Proposal for Introducing more General Aspects of Language in the Teaching of Technical English" en: Lenguas para Fines Específicos: temas fundamentales. Junta de Extremadura. Universidad de Extremadura.
} 
Entre las empresas relacionadas con el transporte de viajeros, sea éste por aire, por ferrocarril, por mar, o por carretera destacan las compañías aéreas, aeropuertos, compañías de transporte ferroviario (die Deutsche Bahn en Alemania y Renfe en España), estaciones de trenes, compañías de autobuses, estaciones de autobuses, puertos, compañías de transporte marítimo o fluvial, etc.

La organización de viajes, distribución y venta es llevada a cabo por las agencias de viajes mayoristas o minoristas y los touroperadores.

4. Las actividades turísticas son organizadas por un amplio abanico de empresas que gestionan desde visitas a museos, monumentos o la explotación de parques temáticos hasta el ejercicio de todo tipo de deportes (deporte de invierno, de riesgo, de élite, etc.) sin olvidarnos de los balnearios o aquellas dedicadas a la información turística , como oficinas de turismo o patronatos.

Esta diversidad de empresas se fundamenta en los múltiples tipos de turismo existentes. Citamos a modo de ejemplo algunos:

turismo cultural / Kulturtourismus

turismo verde y rural / Naturtourismus, Landtourismus

turismo de sol y playa / Strand- oder Sonnentourismus

turismo de invierno / Wintertourismus

turismo de salud / Kurtourismus oder Gesundheitstourismus

turismo de negocios, de incentivos y de congresos / Geschäftstourismus, Incentivetourismus, Kongresstourismus

turismo de estudio / Studientourismus

turismo deportivo / Sporttourismus

Las actividades turísticas derivadas de estos tipos de turismo son innumerables: desde la visita turística cultural, pasando por el turismo de sol y playa, sin olvidarnos del senderismo, trecking, o las estancias en casas rurales, el incipiente turismo de aventuras con modalidades deportivas como el puenting o rafting, las estancias en balnearios, actividades deportivas de todo tipo desde el tenis, al golf o la equitación, desde las visitas derivadas del turismo de congresos hasta las visitas a los parques temáticos, etc.,

Tras esta primera aproximación al mundo turístico podemos constatar con claridad la amplitud y diversidad del sector. El corpus léxico resultante de un análisis en profundidad es igualmente amplio y diverso, pero es delimitable. La cuestión fundamental es ¿cómo didactizar el léxico? ¿qué estrategias metodológicas aplicar para que el aprendizaje de este léxico sea efectivo y pueda ser activado no sólo a nivel pasivo sino también a nivel acti- 
vo $?^{5}$. Podríamos proceder a presentar una selección de palabras recurrentes en cada uno de los campos delimitados, sectores y empresas, por ejemplo:

Hotel

Hotelanlagen:

Hallenbad, Büfettrestaurant, Aufenthaltsraum, Dachterrasse, Solarium, Sauna, Pianobar, Snackbar, Café, Tiefgarage, Parkplatz, Restaurant, Golfplatz, Boutique, Schwimmbad, Tennisplatz, Golfplatz, Souvenirladen, ...

Hotelzimmer:

Schreibtisch, Nachttisch, Papierkorb, Aschenbecher, Telefonapparat, Schrank, Kleiderbügel, Sessel, Fernsehapparat, Spiegel, Vorhang, Bett, Sofa, Handtuch, Kopfkissen, Bettlaken, Klimaanlage, Heizung, ...

Hotelpersonal:

Direktor, Betriebsleiter, Empfangschef, Rezeptionist, Animateur, Restaurantsdirektor, Zimmermädchen, Küchenchef, Personalchef, ...

Hoteltypologie:

Stadthotel, Landhotel, Berghotel, Luxushotel, Kurhotel, Ferienhotel, Terminalhotel, Golfhotel, Seehotel, Seminarhotel, ...

Reisebüro

Typologie:

Reiseveranstalter, Reisagentur, Reisebüro, Reisevermittler, Touroperator, ...

Reiseangebote:

Reise "a forfait", Pauschalreise, Urlaubsreise, Studienreise, Kulturreise, Geschäftsreise, Weltreise, Wochenendreise, Sommerreise, Winterreise, ...

Reiseformen:

Autoreise, Flugreise, Schiffsreise, Bahnreise, Busreise, Gesellschaftsreise, Individualreise, Familienreise, Kulturreise, ...

Reiseleistungen:

Transport, Unterkunft, Verpflegung, Animation, Stadtbesichtigung, Stadtrundfahrt, Transfer zum Hotel, ...

Funktionen:

Quelle zur Reisevermittlung, Information über Reisearten, Information über Flug- und Fahrpläne, Information über Reiseziele, Information über Unterhaltungsmöglichkeiten am Urlaubsort, ...

\footnotetext{
${ }^{5}$ Véase WEISE, G. (1991).
} 
Pero metodológicamente estos listados enciclopédicos son cuestionables. Hecho que se constata a partir de la incursión de lingüistas en la problemática relacionada con los lenguajes específicos a partir de los años 70. Así se llega a la conclusión de que la comunicación específica no viene determinada exclusivamente por un corpus léxico aislado ${ }^{6}$ (Fraas, 1991). La enseñanza especializada requiere una reducción y concentración del léxi$\mathrm{co}^{7}$, pero también la integración de este léxico en un método de aprendizaje global, en el que sea objetivo fundamental la adquisión de destrezas comunicativas que capaciten al discente a activar de forma segura el léxico requerido. Para integrar pues, el corpus léxico en un método global hemos de tener en cuenta en nuestro caso concreto que de cada uno de los sectores, empresas y actividades turísticas mencionadas deriva un gran número de actividades profesionales que dan lugar a las necesidades comunicativas del futuro diplomado. Veamos algunos ejemplos:

En empresas de alojamiento:

dirigir la empresa / einen Betrieb leiten

recibir a huéspedes / Gäste empfangen

informar sobre las instalaciones / die Anlagen beschreiben

reservar habitaciones directamente, por teléfono o por escrito / die Zimmer direkt / telephonisch oder schriftlich buchen

organizar actividades de ocio y tiempo libre / Freizeitsaktivitäten organisieren

En empresas de transporte:

informar sobre horarios y destinos / über Fahr-, Flugzeiten und Reiseziele informieren

reservar plazas / Platzreservierungen vermitteln

En empresas de organización de viajes:

elaborar paquetes turísticos / Pauschalreisen entwerfen

diseñar itinerarios turísticos / Reiserouten entwerfen

En empresas mediadoras de servicios turísticos:

reservar plazas en medios de transportes / in den verschiedenen Verkehrsmitteln Platzreservierungen vermitteln

reserva de plaza en alojamientos / Hotelreservierungen vermitteln

alquiler de coches / Autovermietungen vermitteln

reservar paquetes turísticos / Pauschalreisen buchen und vermitteln

\footnotetext{
${ }^{6}$ En este sentido surge la idea de que existen además del léxico diferencias entre la comunicación específica y la estándar. Véase FRAAS (1991).

${ }^{7}$ Esta tendencia a la reducción y concentración de léxico específico es defendida por ejemplo por STEIGER, J (1993), al analizar aspectos didácticos de otros lenguajes específicos.
} 
informar sobre productos turísticos de alojamiento o transporte / über Unterkunfts- und Transportmöglichkeiten informieren

En empresas de información turística:

informar sobre lugares de interés turístico, horarios de apertura, destinos turísticos, monumentos, visitas turísticas, ... / über Sehenswürdigkeiten, Öffnungszeiten, Urlaubsziele, Monumente, Besichtigungen, ... informieren

Cada una de estas actividades implica el dominio de determinadas situaciones comunicativas recurrentes que, como ya hemos mencionado, estructuralmente no difieren en gran medida de las situaciones comunicativas que necesita el hablante a nivel general en la lengua estándar. Los Diplomados en turismo se enfrentarán a las mismas situaciones comunicativas pero en contextos específicos. Si en la lengua estándar aparecen situaciones comunicativas como la de darse a conocer, expresar o preguntar por los datos personales, a nivel específico el Diplomado en turismo tendrá que preguntar por los datos personales de un cliente en un hotel. Si a nivel general surgen situaciones como la de pedir o dar información, el diplomado en turismo tendrá que dar información sobre los servicios incluidos en un viaje organizado. Si en la lengua estándar es recurrente la situación comunicativa en la que se expresa un deseo, el Diplomado en turismo debe saber expresar el deseo de reservar 10 habitaciones dobles en pensión completa en un hotel de cuatro estrellas o reservar un número determinado de plazas en un avión. Estamos habituados a expresar rechazo, inconformidad o desacuerdo, a nivel específico el egresado debe saber expresar, por ejemplo, su desacuerdo con el sevicio recibido por un touroperador.

Deducimos por tanto, que es el léxico específico inmerso en las situaciones comunicativas específicas el que establece primordialmente la diferencia entre la enseñanza de la lengua estándar y la enseñanza de la lengua con fines específicos en el ámbito turístico. Esta realidad viene a confirmar la postura de muchos especialistas que definen la enseñanza de segundas lenguas con fines específicos como aquella que atiende primordialmente las necesidades comunicativas del discente y apoya el material didáctico en un análisis previo de las mismas. La diferencia viene dada por la presencia de un corpus léxico especializado ${ }^{8}$ pero no por una metodología diferente. Los objetivos son idénticos: se trata de desarrollar en el discente destrezas comunicativas receptoras y productivas, pasivas y activas. Para ello habrá que integrar el léxico necesario en un método. Un método que considere aspectos gramaticales, sintácticos y que haga factible el aprendizaje de este léxico específico.

Analicemos algún ejemplo concreto. Es frecuente que en los grandes hoteles aparezca el problema del llamado "overbooking", una saturación en la reserva de hoteles con el consiguiente problema de tener más huéspedes que habitaciones disponibles. En este caso la solución al problema es "realojar" al cliente en otro hotel:

\footnotetext{
${ }^{8}$ Es destacar que los textos de carácter específico se componen de léxico heterogéneo procedente tanto de la lengua estándar como de la especializada aunque la frontera entre ambos se difumina con facilidad. Véase así el análisis llevado a cabo por Kratzenberger (1991).
} 
Dialog

Kunde: Guten Abend. Mein Name ist Jürgen Orben. Ich habe vor zwei Wochen ein Einzelzimmer für eine Nacht buchen lassen.

Rezeptionist: Ja, ich muss Ihnen aber leider sagen, dass wir voll ausgebucht sind. Ich bitte Sie um Entschuldigung. Wir haben versucht, ein Zimmer für Sie in der Nähe zu finden aber es war unmöglich.

Kunde : Wie bitte? Sie spaßen wohl? Es ist fast Mitternacht und ich habe kein Zimmer? Warum haben Sie mich nicht angerufen? Geben Sie mir bitte das Reklamationsbuch!

Rezeptionist: Beruhigen Sie sich, bitte! Ich bin sicher, dass wir rasch eine Lösung finden werden...

(fünf Minuten später)

Herr Orben, ein Zimmer in einem Hotel unserer Kette ist frei geworden. Es liegt nur fünf Minuten von hier entfernt, ganz in der Nähe. Wir begleiten Sie dahin.

Kunde: Ja, gut! Aber ich möchte trotzdem das Reklamationsbuch, bitte!

Los empleados del hotel, tanto a nivel de dirección como a nivel de recepción deben dar solución no sólo a éste sino a continuos problemas que pueden surgir relacionados con el alojamiento. Se debe aportar al discente la posibilidad de entrar en contacto con el léxico requerido y poder así no sólo y en primera instancia resolver el problema, sino también poder comunicarlo al visitante extranjero. Una posibilidad es mostrar al discente el conflicto en forma de diálogo, como hemos hecho. De esta forma entra en contacto con el léxico de forma contextualizada. Contexto que le facilitará en el futuro activarlo cuando surja una situación análoga. Esta introducción del corpus léxico ha de ir acompañada por un ejercicio sistemático y recurrente del mismo. Más adelante se puede ofrecer al alumno la posibilidad de crear por sí mismo situaciones comunicativas en las que de forma similar debe enfrentarse a la solución de problemas varios. Un ejemplo de este tipo de ejercicios sería el ofrecido en la Figura 1.

El estudiante ha de crear diálogos que den solución al problema planteado. Generalmente es un tipo de ejercicio compartido, en el que un estudiante plantea el problema y otro lo resuelve, reproduciendo así la situación comunicativa real. Aunque limitados por el contexto este tipo de ejercicio creativo ofrece al discente la oportunidad de aportar informaciones, pensamientos y puntos de vista de creación propia tan importantes en la adquisición de destrezas comunicativas (PORTMANN, 1994). Desde un punto de vista didáctico resultan muy adecuados pues contribuyen directamente al carácter comunicativo que debe estar presente en la enseñanza de segundas lenguas. Estos ejemplos responden a una metodología que concibe la enseñanza con fines específicos a partir de modelos de comunicación, como por ejemplo textos en los que se consideren temas, problemas y situaciones que respondan a la comunicación real. Una metodología en la que el léxico específico aparezca integrado en un método global. Una metodología que evite la presentación de léxico de forma independiente, inconexa, sin atender a una progresión lógica en el aprendizaje de la segunda lengua. La enseñanza de segundas lenguas con fines específicos debe ir más allá de unas pocas unidades didácticas relacionadas vagamente 0 , como en muchos otros casos de unas 
pocas unidades didácticas específicas pero desconectadas del método habitual de aprendizaje. Esta metodología a aplicar debe ser la misma que en la enseñanza de segundas lenguas estándar. Pero el carácter específico del léxico potencia la predisposición positiva del alumno al aprendizaje e incrementa su confianza en el éxito, factores esenciales que influyen en el proceso de aprendizaje en general.

Übung: Bilden Sie Dialoge nach dem Muster und lösen Sie diese Probleme!

Guten Tag, kann ich Ihnen helfen?

Guten Tag. Mein Name ist Thomas Wendel, Zimmernummer 110. Ich habe ein kleines Problem. Das Zimmer liegt direkt neben dem Fahrstuhl und ich habe die ganze Nacht mit dem Lärm nicht geschlafen. Ich habe vor, zwei Wochen zu bleiben, aber Sie müssen mir unbedingt ein anderes Zimmer geben.
1.Das Zimmer geht auf den Garten, der Gast wollte ein Zimmer mit Blick auf das Meer.

2. Das Zimmer ist zu klein und zu dunkel. Der Gast möchte ein größeres Zimmer.

3. Es gibt keine Bettwäsche und die Minibar ist leer.

4.Der Fernsehapparat funktioniert nicht.

5. Es gibt keine Kleiderbügel im Schrank.

6. Die Klimaanlage geht nicht, deshalb ist es sehr heiß im Zimmer.

7. Es gibt keine Seife und der Abfall wurde nicht beseitigt.

8. Der Gast hat den Zimmerschlüssel verloren oder im Zimmer vergessen.

9. Er muss früh aufstehen, hat aber keine Uhr.

Figura 1. 


\section{CONCLUSIONES}

A partir de todo lo expuesto podemos establecer las siguientes conclusiones:

En primer lugar resulta evidente la necesidad de abordar la enseñanza del alemán en la Diplomatura de Turismo como específica. ${ }^{9}$

La delimitación del corpus léxico debe partir de un análisis en profundidad del sector turístico. Las actividades profesionales serán las que den lugar a las situaciones comunicativas necesarias.

Estas situaciones comunicativas pondrán de relieve la necesidad de conjugar un léxico de tipo estándar con un léxico de tipo específico, dado que estructuralmente no hay grandes diferencias entre lengua estándar y llamémosle "alemán turístico".

El léxico específico, en este caso turístico ha de estar integrado en un método que facilite el aprendizaje y estimule la motivación del alumno.

El análisis llevado a cabo puede ser aplicado también a otros sectores, no sólo el turístico, con lo cual contribuiríamos de forma muy positiva al desarrollo de métodos que aplicados a la enseñanza de segundas lenguas con fines específicos la potencien.

\section{BIBLIOGRAFÍA.}

EGGERT, S. (1991), Wortschatz ordnen - aber wie? Überlegungen zur Lexiksystematisierung und -differenzierung im Fremdsprachenunterricht Deutsch. Frankfurt. Verlag Peter Lang.

FLUCK, H., (1992), Didaktik der Fachsprachen. Aufgaben und Arbeitsfelder, Konzepte und Perspektiven im Sprachbereich Deutsch. Günter Narr Verlag. Tübingen.

FRAAS, C. (1991), "Sprachpraxis und Terminologiearbeit - Stiefkinder einer Sprachtheorie?”. En: Deutsch als Fremdsprache. 4. Herder-Institut. Leipzig Págs. 201-205.

FREYER, W. (1991), Einführung in die Fremdenverkehrsökonomie, München, R. Oldenbourg Verlag.

KARBE, U. (1990): Didaktische Funktionen von Texten im Fremdsprachenunterricht. En: Deutsch als Fremdsprache. 1. Herder-Institut. Leipzig.. Pág. 13

KOSMA et al. (1998), Betriebsorganisation und Touristik. Linz. Trauner Verlag.

KRETZENBACHER, H. (1991), "Zur Linguistik und Stilistik des wissentschaftlichen Fachworts (1). En: Deutsch als Fremdsprache. 4. Págs. 195-201.

\footnotetext{
${ }^{9}$ Las razones que fundamentan esta postura son, principalmente, la especialización requerida y el corto espacio de tiempo del que disponen docentes y discentes para alcanzar tal competencia lingüística. Así se contempla, de hecho, en las directrices generales propias de la Diplomatura de Turismo, en el que se establece que la enseñanza de las segundas lenguas debe ser impartida con especial aplicación en el sector turístico.
} 
LÖPEZ, A. (1992), Manual de marketing general y de servicios turísticos. Madrid. Síntesis.

MONTANER, J. (1996), Psicosociología del turismo. Madrid. Síntesis.

PASSOV, E.I / KUNTZE, C. (1990).: “Grundlagen einer kommunikativen Methodik für die Befähigung zur fremdsprachigen Kommunikation." En: Deutsch als Fremdsprache. Herder-Institut. Leipzig. 5. Pág. 317.

PASSOV, E.I (1994).: "Kommunikativität als Methodologie des modernen Fremdsprachenunterrichts”. En: Deutsch als Fremdsprache. 5. Herder-Institut. Leipzig. . Pág. 258.

PORTMANN, P. (1994), "Rezeptive und produktive Fertigkeiten". En: Deutsch als Fremdsprache. 2. Herder-Institut. Leipzig. Págs. 96-99.

STEIGER, J (1993), "Fachsprache Deutsch und fachsprachliche Kommunikation". En: Deutsch als Fremdsprache. 2. Herder-Institut. Leipzig. Págs. 88-91.

TRIM, J.L. (1969), "Linguistic considerations in planning courses and in the preparation of teaching materials”. En: Languages for Special Purposes, London, CILT.

WEISE, G. (1991), "Rezeptiver und produktiver Lexikerwerb als Kategorien in der Angewandten Fremdsprachenpsychologie”. En: Deutsch als Fremdsprache. Herder-Institut. Leipzig. 2. Pág. 88. 
